\title{
La pedagogía de la Bauhaus como modelo para la expresión plural en las comunidades artísticas actuales
}

\author{
Milagros García Vázquez
}

Universidad Pontificia de Comillas, mgvazquez@comillas.edu

\begin{abstract}
In 1937 the New Bauhaus in Chicago would take the baton from the original one created by Gropius in 1919. Since then its name has changed, "Chicago School of Design”, "Institute of Design”, until it was integrated into the current "Illinois Institute of Technology". A break between two wars turned into an opportunity for various creators with a common goal, to train other artists to "wish, project and create together the new structure of the future", as Gropius said. Its intended end, imposed by a political regime, found its continuity in a democratic state, where it gave rise not only to that one which was kept institutionally in Illinois, but inspiring others, outside the state structures, examples of a creative alternative inside the current dissent of democratic cultures. The current "Alliance of Artists Communities" is a culmination of all of them. In 1990 the MacArthur Foundation launched a project to enhance the artistic diversity and to promote new creative processes as channels for the improvement of life. Artistic institutions, communities, residences of artists, were invited to the "Special Initiative on Artists", which was the origin of this international consortium of artistic communities. It was a gap of creative plurality and at the same time an occasion to open personal enrichment against the current political dissent.
\end{abstract}

Keywords: Bauhaus, Pedagogy, creativity, dissent, community, alliance, Arts, Politics

\begin{abstract}
Resumen
En el Chicago de 1937 la New Bauhaus tomaría el testigo de la original creada por Gropius en 1919. Su nombre ha variado, "Chicago School of Design», "Institute of Design», hasta integrarse en el «Illinois Institute of Technology» actual. Un paréntesis entre dos guerras fue ocasión para el encuentro de diversos creadores con un objetivo común, formar a otros artistas para «desear, proyectar y crear todos juntos la nueva estructura del futuro», en palabras de Gropius. Su pretendido final, impuesto por un régimen político, encontró su punto y aparte en un Estado democrático, dando pie no sólo a una iniciativa mantenida institucionalmente en Illinois, sino inspirando otras fuera de las estructuras estatales, ejemplos de alternativa creativa en el presente disenso de las culturas democráticas. Una culminación de todas ellas se encuentra en la «Alliance of Artists Communities». En 1990, la Fundación MacArthur lanzaba un proyecto para potenciar la diversidad artística y promover nuevos procesos creativos como cauces en la mejora de la vida. Instituciones artísticas, comunidades, residencias de artistas, fueron convocadas al «Special Initiative on Artists», origen de este consorcio internacional de comunidades artísticas. Una brecha de pluralidad creativa y ocasión de enriquecimiento personal abierta frente al actual disenso político.
\end{abstract}

Palabras clave: Bauhaus, pedagogía, creatividad, disenso, comunidad, alianza, arte, política 
Todo el mundo tenía un mismo lenguaje e idénticas palabras. [...] Dijeron: «Vamos a edificarnos una ciudad y una torre con la cúspide en el cielo, y hagámonos famosos, por si nos desperdigamos por toda la faz de la tierra». Bajó Yahvé a ver la ciudad y la torre que estaban edificando los humanos, y pensó Yahvé: «Todos son un solo pueblo con un mismo lenguaje, y este es el comienzo de su obra. Ahora nada de cuanto se propongan será imposible. Bajemos, pues, y, una vez allí, confundamos su lenguaje, de modo que no se entiendan entre sí». Y desde aquel punto los desperdigó Yahvé por toda la faz de la tierra, y dejaron de edificar la ciudad. Por eso se la llamó Babel, porque allí embrolló Yahvé el lenguaje de todo el mundo (Gn 11, 19).

En la historia de Babel, hombres con lengua común reúnen sus fuerzas para construir una torre y ganar fama en unidad antes de una presumible dispersión. Hay consenso. Sin embargo, el cielo, al que aspiraban sus deseos de gloria, dio lugar a uno de los primeros disensos entre los hombres. Su lengua, única, sufre una ruptura, y el objetivo común se diluye en la dispersión de cada pueblo. El lamento de estos hombres parece escucharse al contemplar el grabado de Gustave Doré La confusión de las lenguas (1865-1868). La creación de su obra no podía continuar sin entendimiento. Entre piedras extraídas con gran esfuerzo, una edificación emergente se transforma en ruinas sin haberse terminado. ¿Podrá cambiar en la historia posterior esta imagen donde la diferencia quiebra la unidad de un trabajo creador? ¿Será posible que esta falta de comunicación se convierta en ocasión para levantar un nuevo edificio con otras aspiraciones a la medida de los artífices? El trabajo de una nueva comunidad de hombres en el siglo XX, con una nueva lengua común, la del arte, revelará si la realidad del grabado se ha tornado más parecida a la visión de Magritte en el cuadro El arte de la conversación (1955). Aquella Confusión de las lenguas surgida entre las piedras, se transforma aquí en los cimientos de una arquitectura original, cuya base es ya una invitación a la renovación, al sueño (Le rêve) de un encuentro, y al pensamiento de hacerlo realidad con piedras nuevas.

Así, en 1919, otra construcción sería el símbolo de una nueva etapa para el arte centroeuropeo. La imagen de una insólita catedral aparecía como frontispicio en la carta de presentación de un nuevo espacio creativo con audaces aspiraciones, para el futuro del arte y de la sociedad. Nacía la Bauhaus, cuyos objetivos fundacionales desempolvaban aquella ambición primigenia. Ahora ya no importan las diferencias, prima la meta común:

«iEl objetivo final de toda actividad plástica es la construcción! [...] Arquitectos, pintores y escultores deben aprender de nuevo a conocer y concebir el carácter multiforme del construir en su totalidad y en sus partes. [...] Deseemos, imaginemos, creemos todos juntos la nueva construcción del futuro, en la que todo, arquitectura, plástica y pintura, confluirá en una sola forma, y que, de la mano de millones de artífices, un día se elevara hacia el cielo como símbolo cristalino de una nueva y venidera fe» (Droste, 1993, 18).

Ese símbolo cristalino quedaba corporeizado en aquel grabado de Lyonel Feininger con la efigie de una nueva catedral, portada de su manifiesto. No en vano, en la Edad Media fueron las catedrales los puntos de convergencia para artistas y artesanos de toda procedencia, especialidad y condición. Gracias a su trabajo en comunidades unidas por el arte de elevar un nuevo edificio, se desarrollaron las ciudades y la sociedad de la época. Todo ello mediante construcciones donde la luz era el signo de cómo las aspiraciones humanas, caídas en la torre de Babel, miraban ahora hacia el cielo con otros ojos. No se trataba ya de tocarlo con la altura de las torres y ganar un nombre, sino de atrapar su esplendor para bajarlo a la tierra y vivir cerca de él. No es extraño que, tras una guerra, hicieran falta sólo unas palabras enérgicas, como las del manifiesto, para excitar en los artistas el deseo de sacar de las trincheras de saco, tierra, lodo y pólvora, la esperanza por ellas sepultada. La misión estaba clara, devolverle a esta virtud su anterior esplendor con las manos de los artistas y artesanos, a través del trabajo común en la belleza de las artes unidas por medio de la arquitectura. Y no como fin en sí mismo, sino con la voluntad de hacer mejor la vida de los hombres, especialmente porque la tarea urge después de haber contemplado tal barbarie y miseria humanas. Que en sus retinas no permanezca demasiado tiempo todo ese horror, que el arte levante enseguida un nuevo templo donde los artistas puedan otra vez encerrar la luz del cielo para convivir con ella, como en las catedrales medievales, parece ser el deseo de aquella innovadora escuela de artistas.

Ya en Inglaterra, el movimiento Arts \& Crafts abría el camino a una iniciativa como la alemana. A mediados del siglo XIX, en respuesta a la uniformidad industrial, teóricos, arquitectos y artesanos unen sus manos para recuperar la impronta 
y valor del trabajo manual y original. Entre ellos William Morris, en sintonía con los modos y maneras medievales, ponía en marcha la escuela y empresa Morris \& Co., en 1875. En ella las prioridades serían no únicamente la formación de artesanos y el rescate del trabajo manual y su ennoblecimiento frente a la industrialización, sino el cultivo del hacer primoroso y de calidad en objetos de todo tipo con el fin de embellecer la vida cotidiana, sacando la belleza de las artes de los museos para llevarla a los hogares. Se trataba de hacer confluir arte y vida en la realidad cotidiana de una sociedad donde la producción impersonal y en serie iba ganando terreno.

Por su parte, ideas semejantes aparecían en Alemania. En 1898 se creaba en Darmstadt la colonia de artistas Mathildenhöhe. Diversos artistas fueron convocados para enseñar nuevas técnicas y modelos a los artesanos de Hessen, con el fin de impulsar el trabajo artesanal en la región. Entre ellos se encontrarían Josef Maria Olbrich y Peter Behrens. El primero, reconocido por su obra dentro de la Secesión vienesa; y el segundo, uno de los fundadores de la Secesión muniquesa. Pero el trabajo más significativo de Behrens llegará en 1907, al convertirse en asesor artístico y proyectista de la AEG en Berlín. Ahora, en el seno del disenso entre artes e industria que había dado lugar a estas iniciativas donde las primeras aspiraban a reencontrar su valor alejándose de la segunda, comienza a vislumbrarse un punto de confluencia, de «conversación». Behrens no sólo diseña la famosa fábrica de turbinas, sino las viviendas de los trabajadores, el logotipo de la empresa, los carteles publicitarios, así como objetos de uso cotidiano vendidos por la marca. Junto a Behrens trabajaría en esta etapa el futuro fundador de la Bauhaus, Gropius, quien se iba empapando de esta nueva forma de unidad entre arte y sociedad.

Behrens participaría igualmente en los proyectos de la Deutsche Werkbund, federación de artistas procedentes de diversas áreas, creada con el fin de llevar a cabo puntualmente trabajos en común. Uno de sus principales impulsores sería el arquitecto Hermann Muthesius, quien, interesado por la actividad del movimiento Arts \& Crafts, recopiló lo aprendido en Inglaterra al respecto en su libro Das englische Haus (1904), para crear en 1907 la Deutsche Werkbund y poner en marcha una nueva línea de trabajo en este camino hacia la unidad entre el arte y la vida. Y es que en dicha aspiración, en su versión inglesa, existía un escollo que Muthesius deseaba salvar. Aunque el proceso artesanal y los buenos materiales suponían una respuesta a la masificación industrial, no se alcanzaba el propósito esencial, llevar el arte a las casas de toda condición social, pues los costos de tales procesos y materias encarecían los precios finales. Así, Muthesius propone la participación activa de los artistas, diseñadores y arquitectos en el mundo de la industria, para dar lugar a objetos prácticos y bellos al mismo tiempo, pero con un coste menor, aprovechando las posibilidades estéticas y de difusión que comporta la producción industrial. Practicidad, diseño y calidad, se convertían en los criterios aplicados a las artes integradas en ámbito doméstico y diario, contando ahora con la ayuda de la industria y la técnica.

Trabajando con Behrens y también siendo miembro de la Deutsche Werkbund, Gropius adquiere completo conocimiento de primera mano sobre todas estas realidades que implican la unidad de las artes en el seno de la sociedad industrial. Así se ganará que en 1915, Henry van de Velde —otro de los adalides de la unión entre utilidad y diseño—, al dejar su puesto Weimar al frente de la Escuela Granducal Sajona de Artes y Oficios, le proponga como sucesor. Inmediatamente después de la Guerra, Gropius funda el espacio donde pondría en marcha sus ideas sobre la conjunción entre arte, artesanía, industria, utilidad y estética de los objetos: nacía la Bauhaus.

Tras la contienda, Gropius toma conciencia de la misión que corresponde en aquellas circunstancias a todo arquitecto como él. Era necesario cambiar su modo de trabajar, lo cual «sólo podría lograrse mediante la formación y preparación de una nueva generación de arquitectos en estrecho contacto con los medios de producción modernos. Una escuela pionera. [...] Requería un equipo completo de personal y ayudantes, que no trabajaran como una orquesta que se inclina ante su director, sino de forma independiente, aunque en cooperación con otros, al servicio de la causa común». Este fue el impulso que hizo posible la Bauhaus, cuyo «objetivo especial era la realización de una arquitectura moderna que, como la naturaleza humana, abarca toda la vida», pero consciente de la realidad social en que debía desenvolverse, en el precipitado desarrollo de la industrialización. Esta integración suponía un reto insoslayable, «evitar la esclavitud del 
hombre por la máquina, protegiendo el hogar y la producción en serie de la anarquía mecánica, llenándola de un fin vivo y con sentido» (Gropius, 1956, 15) ${ }^{1}$.

$\mathrm{El}$ «completo personal» del que hablaba Gropius implicaba la participación plural de artistas y artesanos al mismo nivel de importancia. En definitiva, «lo que la Bauhaus enseñó en la práctica fue la igualdad de derechos de todo tipo de trabajo creativo y su integración lógica dentro del orden mundial moderno». Una conjunción que suponía la reubicación del artista. Para Gropius, la situación de posguerra exigía a los artistas sumergirse en el mundo real, no podían seguir en una suerte de «parnaso» al margen de las realidades y necesidades del resto de la sociedad. Por ello, otro de los objetivos de la Bauhaus sería el de «sacudir al artista creativo de su extrañeza en el mundo y restaurar su relación con el mundo del trabajo real». Pero además, esta preocupación por la reaparición del artista en la sociedad y sus tareas, al transformarse de teoría en acción, buscaba relajar la incipiente actitud mercantilista del mundo industrializado. O en palabras de Gropius, se trataba de lograr una unidad entre todo tipo de creación «diametralmente opuesta a la idea del "arte por el arte" y a la filosofía aún más peligrosa surgida de ella, la del negocio como fin en sí mismo» (Gropius, 1955, 15-16).

Dadas estas perspectivas, resulta lógico el formato al que el arquitecto recurre para alcanzar esta coalición entre creación artística y sociedad. Propone el modelo, actualizado, de las «Hütte», en alemán, simplificación de la palabra original «Bauhütte», institución medieval donde se congregaban las diversas artesanías partícipes en la edificación de las catedrales. Gropius tomará de ellas su modo de trabajo, según el cual «numerosos artistas, arquitectos, escultores y artesanos reunidos en todos sus grados, animados por un mismo espíritu y por respeto hacia la idea común a la que servían y cuyo sentido entendían, supieron integrar el trabajo conjunto asignado dentro su tarea individual» (Wingler, 2002, 30). Este sería el ejemplo de referencia para Gropius, incluso para dar el nombre a la propia Bauhaus, donde «Hütte», es sustituido por «Haus», la cabaña medieval por casa del siglo XX.

Su primera sede fue Weimar. Los profesores ahora se llamarían maestros, y los alumnos aprendices. Éstos serían formados en arquitectura, escultura o pintura y en todas sus derivadas artesanales, talla, cerámica, tejidos, diseño de jardines y muebles, cantería, vidriado, pasando además por la Historia del Arte, la anatomía o las teorías químicas sobre el color. A esta enseñanza integral y colaborativa se unía la convivencia lúdica en diversos festivales, entre los que destacan las representaciones teatrales, como la puesta en escena de Das Triadisches Ballet creado por Oskar Schlemmer, en 1923, o su primera exposición pública celebrada en el mismo año. En ambos ejemplos todos los elementos eran realizados por los estudiantes, la convivencia era, por tanto, intensa, tanto entre los aprendices, como entre éstos y los maestros. Se trataba de una verdadera comunidad artística, alimentada con teoría y práctica, con ideas sobre el papel y realidades terminadas desplegadas en todos los ámbitos de la creación. Parecía realizarse el ideal propuesto por Gropius en el manifiesto fundacional, la misión de la Bauhaus: «preparar a arquitectos, pintores y escultores de todas las categorías, según sus capacidades, para que se conviertan en hábiles artesanos o en artistas creadores independientes y fundar una comunidad de trabajo formada por maestros y aprendices capaz de construir unitariamente obras completas —estructura, cuerpo, decoración, mobiliario - a partir de un espíritu común» (Droste, 1993, 18) . Es lo que Gropius llamaba «la gran construcción».

Feininger, Johannes Itten, Paul Klee, Lothar Schreyer, Kandinsky, Herbert Bayer, Marcel Breuer, Gunta Stölz, László Moholy-Nagy, fueron algunos de los maestros. Cada uno se ocupaba de un área, aunque destacó por su singularidad el curso preliminar, dirigido desde 1919 por Itten y más tarde por Moholy-Nagy (1923-1928) y Josef Albers (1923-1933). El propósito de este curso era el de cribar lo aprendido por los estudiantes antes de su ingreso, alejarles del modelo académico de enseñanza artística, así como el descubrimiento, mediante la práctica, de la verdadera rama de su vocación artística, la liberación de sus destrezas y el enfrentamiento con los problemas esenciales de la creación. Dichas actividades abarcaban desde los ejercicios gimnásticos, para aprender acerca del ritmo y el movimiento, hasta la enseñanza sobre formas, colores, texturas, materiales o contrastes entre sombras y luces. Tras la marcha de Itten, Moholy-Nagy y Albers, lo variaron en función de sus objetivos y criterios pedagógicos. El primero, artista experimentador con una importante faceta teórica, potenció las posibilidades estéticas de las nuevas vías abiertas por la técnica. El segundo,

Todas las citas extraídas de textos escritos en alemán han sido traducidas por la autora de este artículo. 
extraordinariamente polifacético, se preocupó por favorecer la relación directa de los aprendices con los materiales, dejando de lado la teoría y la historia, procurando remover y despertar sus capacidades creativas con pruebas constantes, invitándoles a probar instrumentos o técnicas aún no utilizados.

Realmente, la enseñanza artística propuesta por la Bauhaus más que crear un nuevo modelo, catalizaba las nacientes tendencias presentes a comienzos de siglo orientadas hacia una renovación radical del aprendizaje y de la concepción del arte. Así lo dejaba claro Gropius cuando publicó en 1924 una relación ${ }^{2}$ recogiendo los nombres de quienes, antes que él, habían reflexionado sobre esta necesidad de cambio en la educación artística, considerando la integración de las artes entre sí y de éstas con los avances tecnológicos.

En esta semblanza sobre las bases pedagógicas de la Bauhaus se dejan reconocer dos líneas de esfuerzo que ya definen un modo de trabajo artístico plural viable y sostenible mediante objetivos consensuados claros. Por un lado, la interacción de todas las ramas artísticas y artesanales, entre ellas y también dentro de las propias capacidades creativas de cada aprendiz, aun especializado en una, se buscaba dotarle de conocimiento y práctica en el resto. El arte era entendido como unidad, como un cuerpo con muchos miembros, cada uno con una función, pero integrados en un todo y con una misión común. Por otra parte, este deseo de abarcar las posibilidades creativas humanas contenía implícita la voluntad de integrar el arte en todas las esferas de la vida, y lograr hacerlo descender del pedestal en donde se le contemplaba, concediéndole un papel cotidiano y no excepcional. Y todo ello, con la intención de salvar al hombre de la esclavitud de la tecnología y la mecanización, humanizando las nuevas técnicas al convertirlas en instrumentos que ayudan a la creatividad humana, en lugar de tomarlas por ingenios de los que depender.

Pero al llegar 1933, esta ebullición de creatividad y trabajo cesó. En 1924 un cambio de gobierno en el Parlamento regional de Turingia provocó el cierre de la Bauhaus por falta de ayudas económicas. Dessau sería su nueva sede desde 1925. En 1928 Gropius es sucedido por Hannes Meyer cuyas prioridades van a ser la practicidad y el compromiso social, y su foco de atención principal la arquitectura, quedando relegada a un segundo plano la formación en el resto de las artes. En 1930 es sustituido por Mies van der Rohe, y en 1932, con la derrota de los socialdemócratas en Dessau, se hace necesaria una nueva sede. Sobrevivirá en Berlín unos meses, hasta que su actividad fue sumada a la lista de aquel «arte degenerado y bolchevique» perseguido por el régimen nazi, por lo que Gestapo forzó el cierre de sus puertas el 20 de julio de 1933.

Moholy-Nagy había abandonado la Bauhaus en 1928 y trabajado de forma independiente desde entonces. En 1937, la Association of Arts and Industries (AAI) de Chicago, le ofrecía, por recomendación de Gropius, el puesto de director en una nueva escuela de diseño. La idea era crear una escuela siguiendo el modelo de la Bauhaus europea, habilitando un lugar donde formación, artes e industria encontraran su espacio de colaboración y crecimiento, contando con el respaldo de importantes empresarios. Así pues, si en Alemania el consenso de los artistas a favor de la pluralidad de las artes, incorporada a las posibilidades brindadas por la técnica, encontraba su disolución con el ascenso al poder del pensamiento único y de la censura de las vías creativas ofrecidas por el arte, aquella coalición daba ahora con nueva tierra fértil. Recomenzaba el camino en un país en que seguía siendo posible encontrar una unidad actuante, dentro de la diferencia, sin interferencias políticas. Moholy-Naghy daba su sí a la propuesta, así se lo contaba a su esposa Sibyl: «Este país y sus habitantes tienen algo de inacabado que me fascina, urge completarlo. Aquí todo me parece posible. El carácter definitivo y paralizante de la catástrofe europea ha quedado atrás. Me encanta la atmósfera de lo nuevo y de la expectativa que aquí he encontrado» (Sibyl, 1972, 123).

En septiembre de 1937, Moholy-Nagy presentaba en público los objetivos de la Nueva Bauhaus de Chicago. La tarea de la escuela sería la de formar al «técnico del arte», con experiencia en «fibras sintéticas, moda, teñido y estampados de telas, diseños de papel tapiz, murales, uso de esmaltes, pinturas, pinturas en aerosol, [...], tipografía, diseño, fotografía comercial y de retratos, fotomicrografía, color y películas de alta resistencia, arte comercial en vallas publicitarias, [...]

2 Dicha lista aparece recogida en una recopilación de artículos en prensa sobre la Bauhaus aparecidos en diversas publicaciones alemanas desde 1920 a 1924: Pressestimmen (Auszüge) für Staatliche Bauhaus Weimar (1924). Weimar: R. Wagner Sohn, pp. 71 y ss. 
escenarios, equipamiento de escaparates y tiendas, arquitectura de exposiciones y todas las estructuras arquitectónicas, desde el bungalow prefabricado, hasta la fábrica», y además «con todos los materiales: piedra, vidrio, metal, arcilla y plásticos». Y es que según Moholy-Nagy, «en el futuro nunca podremos mirar a un individuo sin referirnos al todo» (Sibyl, 1972, 127). El plan de estudios incluía igualmente biotécnica, biología, química, física, matemáticas, geometría, psicología, filosofía y sociología. Diferentes sociedades e investigadores americanos reconocían no haber contado hasta entonces con una institución que se esforzase por sintetizar el saber en todas las áreas del conocimiento. La acelerada aparición de abundantes descubrimientos técnicos desde la Revolución Industrial, les había hecho perder la capacidad de darles una unidad, desarrollando trabajos cada vez más especializados. Para devolver a la vida ese equilibrio perdido, se veían urgidos a unificar las posibilidades técnicas generadas en los diferentes campos de la actividad humana, y optimizar su eficacia en la práctica colaborativa podría ser la solución.

Sin embargo, la elevada implicación empresarial en el funcionamiento de la Escuela hizo que las cuestiones económicas fueran prioridad, a lo que se añadieron las deudas y la crisis económica de 1938 en Estados Unidos. El director de la AAI comunicaba a Moholy-Nagy el cierre de la Escuela a finales de año. Sintiéndose responsable del futuro de los alumnos a los que había comenzado a formar, tomaría la resolución de crear su propia escuela. En 1939 abría la Escuela de Diseño (School of Design), situada también Chicago. Esta vez no habría consejo administrativo, pues según Moholy, el éxito de una escuela no puede residir en el mundo de las finanzas, sino en hombres que respalden moralmente sus objetivos y los compartan con el director. Entre ellos se encontraron J. Dewey, el propio Gropius, Joseph Hudnunt, proveniente de la Escuela de Diseño de Harvard, o Alfred H. Barr, primer director del MoMa. En el discurso inaugural, Moholy definía las líneas de la escuela:

«Se trata menos de una escuela que de un laboratorio, donde no contarán los resultados, sino los caminos a través de los cuales se lograrán esos resultados. [...] Ustedes son la medida de nuestros esfuerzos educativos como seres humanos totales, no por su capacidad como futuros diseñadores de muebles, artistas gráficos, fotógrafos o conferenciantes. Sus cerebros y sus manos, sus emociones y su salud son parte del proceso de trabajo. No piensen que pueden perfeccionar una de estas partes si descuidan otra, eso destruiría la integridad de sus capacidades. De ustedes depende cómo den forma lo que se encuentra latente en su interior. Si logran formar comunidades de trabajo unos con otros, entonces, los resultados a partir de la unión de sus fuerzas superarán a la escuela técnica mejor equipada» (Sibyl, 1972, 141).

Las complejas aspiraciones de la «Nueva Bahuaus», fueron sustituidas por el retorno a los conceptos elementales de la original alemana, al tratamiento directo de los problemas básicos en materiales, luces, volúmenes, color y espacio. Incluso se abrieron clases y talleres para niños. Y aunque no faltaron empresas benefactoras para la escuela, a partir de la participación de Estados Unidos en la II Guerra Mundial, se intensifican las dificultades económicas. El número de alumnos descendió y los precios de los materiales subieron. Muestra de que a Moholy-Nagy le preocupaba la verdadera integración y valiosa aportación de las creaciones y enseñanzas de la escuela en la sociedad fueron los nuevos ámbitos que descubría en aquellos años. La aplicación más querida por Moholy fue el desarrollo de ejercicios de coordinación, mediante la creación de formas a partir de materiales conocidos, como terapia de rehabilitación para los mutilados de guerra. Una faceta de la creación artística que era también enseñada en el centro a los estudiantes.

Las dificultades económicas prosiguieron a lo largo de los años que duró la contienda. «Fue el espíritu de cooperación lo que unió a los maestros y estudiantes», dijo Moholy en una ocasión. En tiempo de guerra el significado de la escuela también entraba en crisis.

«Estudiantes y maestros se preguntaban si nuestro trabajo en tiempos de guerra no era un lujo. [...] Ciertamente, es un gran privilegio, $[\ldots]$ pero este privilegio les es otorgado por la sociedad, y es una inversión para el beneficio futuro de los hombres. De ustedes y de sus serios esfuerzos depende el futuro progreso de la educación. No importa si hacen muelles o sillas de madera, si diseñan casas o carteles, o si trabajan con veteranos y niños. [...] La democracia está basada en el intercambio de 
igualdades. Y aquellos a quienes se les ha permitido desarrollar sus mayores talentos, tienen el deber de emplear un día sus habilidades creativas para la existencia armónica y productiva de una nueva generación» (Sibyl, 1972: 156).

El éxito conseguido, a pesar de todo, llamó la atención en 1944 de un conjunto de empresarios, quienes ofrecían el soporte necesario para la supervivencia de la Escuela. Ahora pasaría a llamarse «Instituto de Diseño», tendría el rango de institución equiparable al de las universidades, y debería convertirse en un lugar rentable donde educar y formar futuros trabajadores. Las convicciones de Moholy, diametralmente opuestas a la mentalidad empresarial americana, se mantenían incólumes. A pesar de la presión, su programa seguía respondiendo a sus ideales, en beneficio, decía, de la juventud estadounidense que «valore la integración creativa más que las habilidades aprendidas rápidamente o la obtención de un trabajo lucrativo» (Sibyl, 1972: 179).

La lucha de Moholy llegaba al final. El diagnóstico: leucemia. Aún así, continuaba al frente del ahora Instituto, impartiendo cursos y pendiente de los alumnos. Al regresar de una de sus clases de pintura, ingresaba en el hospital, y fallecía unos días después, el 24 de noviembre de 1946. Gropius recomendaría como sucesor a Serge Chermayeff, arquitecto y diseñador industrial, quien ocuparía el cargo de director del Instituto de Diseño hasta 1951, siendo el responsable de su integración en el actual Illinois Institute of Technology (IIT), en la misma ciudad de Chicago.

El modelo de la Bauhaus, prolongado en Chicago, como centro de creatividad artística honesta puesta al servicio de la sociedad, tuvo una amplia repercusión desde el punto de vista pedagógico en el resto de Estados Unidos, más de lo que lo sería en Europa, convertida desde 1933 en tierra hostil para las nuevas iniciativas artísticas del siglo. Por otra parte, muchos de los alumnos y profesores de la Bauhaus, al igual que Moholy-Nagy y Gropius, se vieron inmersos en una diáspora artística debido a esa negación de posibilidades.

Antes de la llegada de los Bauhäusler existían en Estados Unidos ecos del Art\&Crafts inglés, como en el caso de la Prairie School, promovida por Frank Lloyd Wright a finales del siglo XIX en Chicago, donde precisamente se crearía en 1922 la Association of Arts and Industries, origen de la New Bauhaus. Ya con resonancias bauhausianas, el editor George G. Booth, creaba en 1935 la Cranbrook Academy of Art (Michigan), dedicada a la enseñanza de arquitectura, arte y diseño. El arquitecto E. Saarinen sería su primer director. Albers había emigrado ya a Estados Unidos en 1933 y encontrará su lugar en el Black Mountain College, centro de estudios interdisciplinar fundado por el humanista John A. Rice en Carolina del Norte. En la Universidad de Harvard, la Escuela de Diseño aparecía en 1936, y sería dirigida por Gropius, quien contó con el apoyo además de otro exiliado de la Bauhaus, M. Breuer. En 1939, inspirados por la actividad de estas escuelas, el arquitecto Gordon Herr y su esposa, la escritora Jane Herr, deciden crear una colonia de artistas en California, conocida como Pond Farm, activa hasta 1953. Uno de los empresarios que respaldaron los esfuerzos de Moholy-Nagy, Walter Paepcke, fundaría en Aspen en 1950 un Instituto de Estudios Humanísticos, Aspen Institute. En este caso el proyecto fue concebido pensando en los empresarios y en el cultivo en ellos de la sensibilidad hacia las posibilidades humanizadoras de las artes en medio del fragor de la utilidad y productividad modernas.

Acercándonos a la actualidad, otro de aquellos exitosos empresarios americanos, John D. MacArthur, creaba en 1970 en Chicago la MacArthur Foundation, con la misión de promover «personas creativas, instituciones eficaces y redes de influencia para construir un mundo más justo, verde y pacífico», tal como reza su lema. En el marco de su programa de becas de investigación destinadas a personas «creativas e inspiradoras», desde artistas, químicos, poetas, músicos, escritores, sociólogos, coreógrafos, neurocientíficos, abogados o compositores, una de sus principales iniciativas llegaba en 1990. Dieciocho grupos — colonias, comunidades y residencias - de artistas eran convocados y dotados económicamente para formar parte de un encuentro en que conocerse, compartir ideas y generar proyectos. Aquella cita, Special Initiative on Artists, mantenida en Febrero de 1991 en el Atlantic Center for the Arts (Florida), y planteada como evento puntual, daría lugar a la hoy conocida como Alliance of Artists Communities. El primer encuentro como tal de este consorcio internacional de artistas tuvo lugar en San Francisco en 1992. A La Alianza pertenecen residencias de artistas de todo el mundo, y como institución mantiene el contacto entre ellas en pequeñas redes con objetivos comunes, las recoge en completos directorios accesibles para artistas interesados en participar en alguna de ellas según sus inquietudes; promueve, impulsa y apoya los programas de las residencias miembros de la Alianza; ofrece servicios de consultoría y 
asesoramiento a las comunidades que lo soliciten, así como formación específica. Cada año celebra la Convención Anual, importante y multitudinario encuentro de comunidades artísticas, donde se dan cita artistas, profesores de arte, directores de escuelas artísticas y miembros de comunidades procedentes de todo el mundo. Entre sus temas, como preocupación constante, se encuentra siempre el papel del artista en la sociedad actual.

Así pues, el acercamiento al itinerario histórico y pedagógico de la Bauhaus nos permite constatar que es posible hoy $E l$ arte de la conversación invocado por Magritte. El arte ofrece, en el marco de la desconfianza hacia las estructuras democráticas — nuestra «Babel» actual—, un espacio en el que la pluralidad aún es positiva y creativa. La afirmación de esta realidad se distingue en el itinerario aquí trazado. El disenso entre artes e industria, revitalizaba a las primeras con el movimiento Art\&Crafts. Después, el desacuerdo se resolvía haciéndose viable la integración entre creatividad, industria, estética y vida gracias a la Bauhaus. Llegaba luego un fuerte disenso, ahora entre arte y política, el nazismo desterraba la pluralidad creativa. Estados Unidos se abría como nueva tierra prometida para continuar el camino. Y, de nuevo, el desacuerdo, aquí entre economía y creación, escollo salvado gracias a las nuevas comunidades de artistas y a la supervivencia del apoyo filantrópico. Mientras los políticos se enfrentan, las artes siguen «conversando», y nos recuerdan que, ante la dispersión «babilónica» presente, existe aún la esperanza en «la construcción del futuro».

\section{Referencias}

Alliance Of ARTISTS Communities. History. 〈https://artistcommunities.org/history> [Consulta: 26 de junio de 2019]

ASPEN InSTITUTE. A Brief History of the Aspen Institute. 〈https://www.aspeninstitute.org/about/heritage/> [Consulta: 29 de agosto de 2019]

Biundo, CH. et al. (1994). Bauhaus Ideen. 1919-1994. Bibliografie und Beitrage zur Rezeption des Bauhausgedankens. Berlín: Dietrich Reimer Verlag.

Black Mountain College. Our Mission Statement. 〈http://www.blackmountaincollege.org/about/> [Consulta: 26 de agosto de 2019]

CRANBROOK ACADEMY OF ART. How it all began. <https://cranbrookart.edu/about/history/> [Consulta: 26 de agosto de 2019]

Droste, M. (1993). Bauhaus Archiv. 1919-1933. Colonia: Benedikt-Taschen Verlag.

GROPIUS, W. (1956). Architektur. Frankfurt/M-Hamburgo: Fischer Bücherei.

ILlinOIS InSTITUTE Of DeSIGN. The New Bauhaus. 〈https://id.iit.edu/new-bauhaus/> [Consulta: 23 de junio de 2019]

MACARTHUR Foundation. About us. <https://www.macfound.org/about/> [Consulta: 26 junio de 2019]

Moholy-NaGy, S. (1972). László Moholy-Nagy. Mainz: Florian Kupferberg Verlag.

Neuman, E. (1996). Bauhaus und Bauhäusler. Erinnerungen und Bekenntnisse. Colonia: DuMont Verlag.

Todd Cook, M., Romano, J. Building a New Foundation: Adapting Bauhaus Pedagogy for the Future of Design. <https://id.iit.edu/wpcontent/uploads/2019/06/mediaID-Bauhaus-Newsletter.pdf> [Consulta: 1 de septiembre de 2019]

WIECK, R., (2007). La pedagogía de la Bauhaus. Madrid: Alianza.

Wingler, H. M., (2002). Das Bauhaus. Weimar, Dessau, Berlin, 1919-1933. Colonia: DuMont Literatur und Kunst Verlag. 